\title{
Numerical solution of nonlinear two-dimensional Volterra integral equation of the second kind in the reproducing kernel space
}

\author{
A. Fazli ${ }^{1} \cdot$ T. Allahviranloo ${ }^{1} \cdot$ Sh. Javadi ${ }^{2}$
}

Received: 9 July 2016/Accepted: 6 March 2017 / Published online: 28 March 2017

(c) The Author(s) 2017. This article is an open access publication

\begin{abstract}
In this article, an effective method is given to solve nonlinear two-dimensional Volterra integral equations of the second kind. First, we find the solution of integral equation in terms of reproducing kernel functions in series, then by truncating the series an approximate solution obtained. In addition, the calculation of Fourier coefficients solution of the integral equation in terms of reproducing kernel functions is notable. Numerical examples are presented, and their results are compared with the analytical solution to demonstrate the validity and applicability of the method.
\end{abstract}

Keywords Two dimentional Volterra integral equation of the second kind · Reproducing kernel · Fourier coefficients

\section{Introduction}

Many problems in engineering and mechanics appear to be two-dimensional integral equations. For example, it is usually necessary to solve Fredholm integral equations in the calculation of plasma physics [1]. Mckee et al. [2] revealed that a class of nonlinear telegraph equations is equivalent to two-dimensional Volterra integral equations. Graham [3] showed that two-dimensional Ferdholm integral equation used in solving the problems of electrical engineering. Some other applications of two-dimensional integral equations can be found in [2, 4].

T. Allahviranloo

allahviranloo@yahoo.com

1 Department of Mathematic, Science and Research Branch, Islamic Azad University, Tehran, Iran

2 Department of Mathematic, Kharazmi University, Tehran, Iran
In this paper, we consider the nonlinear two-dimensional Volterra integral equation of the second kinds as follows:

$u(x, t)=f(x, t)+F u(x, t) \quad(x, t) \in D=[a, b] \times[c, d]$

where

$F u(x, t)=\lambda \int_{c}^{t} \int_{a}^{x} K(x, t, y, z) N(u(y, z)) \mathrm{d} y \mathrm{~d} z$.

Here, $u(x, t)$ is unknown function and $u(x, t), f(x, t) \in$ $W_{2}^{(1,1)}(D), N($.$) the continues terms in W_{2}^{(1,1)}(D), W_{2}^{(1,1)}(D)$ is a reproducing kernel space. Little numerical methods for nonlinear integral equations are written. In this context, the methods used are the block-by-block method [5], blockpulse functions [6], and rationalized Harr function [7].

In this article, we obtain presentation of exact solution nonlinear two-dimensional Volterra integral equation of the second kind in reproducing kernel space, and then, approximate solution is obtained by cutting series. Of course, coefficients of the series on presentation of exact solution obtained with a numerical calculation. The error of the approximate solution is monotone deceasing in the sense of $\|\cdot\|_{W_{2}^{(1,1)}}$.

\section{The fundamental principles of the method}

Below are some definitions and theorems that we used in the next sections.

Definition 2.1 [9] The function space $W_{2}^{1}[a, b]$ is defined as follows:

$$
\begin{gathered}
W_{2}^{1}[a, b]=\{f(x) \mid f(x) \text { is absolutely continuous, } \\
\left.f^{\prime}(x) \in L^{2}[a, b], x \in[a, b]\right\} .
\end{gathered}
$$


Definition 2.2 [9] The inner product and norm in the function space $W_{2}^{1}[a, b]$ are defined as follows:

For any functions $f(x), g(x) \in W_{2}^{1}[a, b]$,

$$
\langle f, g\rangle_{W_{2}^{1}}=f(a) g(a)+\int_{a}^{b} f(x) g(x) \mathrm{d} x
$$

and

$$
\|f\|_{W_{2}^{1}}=\sqrt{\langle f, f\rangle_{W_{2}^{1}}}
$$

It is easy to prove that $W_{2}^{1}[a, b]$ is an inner space. At [9] prove that function space $W_{2}^{1}[a, b]$ is a Hilbert space and also it is a reproducing kernel space.

Suppose $R_{y}(x)$ is the reproducing kernel of $W_{2}^{1}[a, b]$; in this case, we have for any $f(x) \in W_{2}^{1}[a, b]$ :

$$
\left\langle f(x), R_{y}(x)\right\rangle_{W_{2}^{1}}=f(y) .
$$

With calculation, we obtain that

$$
R_{y}(x)=\left\{\begin{array}{lll}
1-a+x & \text { if } & x \leq y \\
1-a+y & \text { if } & x>y
\end{array}\right.
$$

Let set $D=[a, b] \times[c, d] \subset \mathcal{R}^{2}$.

Definition 2.3 [9] The binary function space is defined as $W_{2}^{(1,1)}(D)=\{f(x, y) \mid f(x, y)$ is completely continuous in

$$
\left.D, \frac{\partial^{2} f(x, y)}{\partial x \partial y} \in L^{2}(D)\right\} \text {. }
$$

Definition 2.4 [9] The inner product and norm in $W_{2}^{(1,1)}(D)$ is defined as follows:

$$
\begin{aligned}
\langle f(x, y), g(x, y)\rangle_{W_{2}^{(1,1)}} & =\int_{c}^{d} \frac{\partial f(a, y)}{\partial y} \frac{\partial g(a, y)}{\partial y} \mathrm{~d} y \\
& +\langle f(x, c), g(x, c)\rangle_{W_{2}^{1}}
\end{aligned}
$$

and

$$
\|f\|_{W_{2}^{(1,1)}}=\sqrt{\langle f(x, y), f(x, y)\rangle_{W_{2}^{(1,1)}}} .
$$

It is easy to prove that $W_{2}^{(1,1)}(D)$ is an inner space. At [9] prove that function space $W_{2}^{(1,1)}(D)$ is a Hilbert space and also it is a reproducing kernel space which has reproduced kernel:

$K_{(\xi, \eta)}(x, y)=R_{\xi}(x) Q_{\eta}(y)$ where $R_{\xi}(x), Q_{\eta}(y)$ are the reproducing kernels of $W_{2}^{1}[a, b]$ and $W_{2}^{1}[c, d]$, respectively.

Therefore, for each $f(x, y) \in W_{2}^{(1,1)}(D)$

$\left\langle f(x, y), K_{(\xi, \eta)}(x, y)\right\rangle=f(\xi, \eta)$.

Thus, if $K_{(y, z)}(x, t)$ is reproducing kernel of $W_{2}^{(1,1)}(D)$, then $K_{(y, z)}(x, t)=R_{y}(x) Q_{z}(t)$, where $R_{y}(x)$ and $Q_{z}(t)$ are reproducing kernels in $W_{2}^{1}[a, b]$ and $W_{2}^{1}[c, d]$, respectively.

We will display the solution of Eq. (1) in reproducing kernel space $W_{2}^{(1,1)}(D)$ for this purpose let $\phi_{i}(x, t)=K_{\left(x_{i}, t_{i}\right)}(x, t)$, where $\left\{\left(x_{i}, t_{i}\right)\right\}_{i=1}^{\infty}$ is dense in region $D$. From the property of the reproducing kernel, we have

$$
\begin{aligned}
& \left\langle u(x, t), \phi_{i}(x, t)\right\rangle_{W_{2}^{(1,1)}(D)}=\left\langle u(x, t), K_{\left(x_{i}, t_{i}\right)}(x, t)\right\rangle_{W_{2}^{(1,1)}(D)} \\
& =u\left(x_{i}, t_{i}\right) .
\end{aligned}
$$

Theorem 2.1 If $\left\{\left(x_{i}, t_{i}\right)\right\}_{i=1}^{\infty}$ is dense in the region $D$, then $\left\{\phi_{i}(x, t)\right\}_{i=1}^{\infty}$ is the complete function system of $W_{2}^{(1,1)}(D)$.

Proof Suppose $u(x, t) \in W_{2}^{(1,1)}(D)$ if $\left\langle u(x, t), \phi_{i}(x, t)\right\rangle_{W_{2}^{(1,1)}(D)}=u\left(x_{i}, t_{i}\right)=0 \quad(i=1,2, \ldots)$.

Then, we have $u(x, t)=0$ from the density of $\left\{\left(x_{i}, t_{i}\right)\right\}_{i=1}^{\infty}$ and continuity of $u(x, t)$.

By applying Gram-Schmidt orthonormalization process for $\left\{\phi_{i}(x, t)\right\}_{i=1}^{\infty}$

$$
\bar{\phi}_{i}(x, t)=\sum_{k=1}^{i} \alpha_{i k} \phi_{k}(x, t)
$$

where $\alpha_{i k}$ are coefficients of Gram-Schmidt orthonormalization and $\left\{\bar{\phi}_{i}(x, t)\right\}_{i=1}^{\infty}$ is a normal orthogonal basis of $W_{2}^{(1,1)}(D)[9]$.

Now, the following theorem is obtained.

Theorem 2.2 We assume $\left\{\left(x_{i}, t_{i}\right)\right\}_{i=1}^{\infty}$ be dense in region D. If Eq. (1) has a unique solution, then it is as follows:

$$
u(x, t)=\sum_{i=1}^{\infty} \sum_{k=1}^{i} \alpha_{i k}\left(f\left(x_{k}, t_{k}\right)+F u\left(x_{k}, t_{k}\right)\right) \bar{\phi}_{i}(x, t) .
$$

Proof Suppose $u(x, t)$ is the solution of Eq. (1). From Theorem 2.1 and since $\left\{\bar{\phi}_{i}(x, t)\right\}_{i=1}^{\infty}$ is a normal orthogonal basis of $W_{2}^{(1,1)}(D)$, therefore, we can write

$$
\begin{aligned}
& u(x, t)=\sum_{i=1}^{\infty}\left\langle u(x, t), \bar{\phi}_{i}(x, t)\right\rangle_{W_{2}^{(1,1)}} \bar{\phi}_{i}(x, t) \\
& =\sum_{i=1}^{\infty} \sum_{k=1}^{i} \alpha_{i k}\left\langle u(x, t), \phi_{k}(x, t)\right\rangle_{W_{2}^{(1,1)}} \bar{\phi}_{i}(x, t)
\end{aligned}
$$


$=\sum_{i=1}^{\infty} \sum_{k=1}^{i} \alpha_{i k}\left\langle f(x, t)+F u(x, t), \phi_{k}(x, t)\right\rangle_{W_{2}^{(1,1)}} \bar{\phi}_{i}(x, t)$

$=\sum_{i=1}^{\infty} \sum_{k=1}^{i} \alpha_{i k}\left(f\left(x_{k}, t_{k}\right)+F u\left(x_{k}, t_{k}\right)\right) \bar{\phi}_{i}(x, t)$.

The proof is complete [9].

\section{Implementations of the method}

In this section, a method will be presented to calculate the solution (2) of the Eq. (1). To this end rewrite (2) as

$u(x, t)=\sum_{i=1}^{\infty} A_{i} \bar{\phi}_{i}(x, t)$

where

$A_{i}=\sum_{k=1}^{i} \alpha_{i k}\left(f\left(x_{k}, t_{k}\right)+F u\left(x_{k}, t_{k}\right)\right)$

$A_{i}$ is unknown, because $u(x, t)$ is an unknown function. $A_{i}$ with a numerical calculation can be approximated by known $B_{i}$. For this purpose, put initial function $u_{1}(x, t)=$ $f(x, t)$ and $n$-term approximation for $u(x, t)$ is defined as follows:

$u_{n+1}(x, t)=\sum_{i=1}^{n} B_{i} \bar{\phi}_{i}(x, t)$

where

$$
\begin{aligned}
& B_{1}=\alpha_{11}\left(f\left(x_{1}, t_{1}\right)+F u_{1}\left(x_{1}, t_{1}\right)\right) \\
& u_{2}(x, t)=B_{1} \bar{\phi}_{1}(x, t) \\
& B_{2}=\sum_{k=1}^{2} \alpha_{n k}\left(f\left(x_{k}, t_{k}\right)+F u_{2}\left(x_{k}, t_{k}\right)\right) \\
& u_{3}(x, t)=B_{1} \bar{\phi}_{1}(x, t)+B_{2} \bar{\phi}_{2}(x, t) \\
& \ldots \\
& B_{n}=\sum_{k=1}^{n} \alpha_{n k}\left(f\left(x_{k}, t_{k}\right)+F u\left(x_{k}, t_{k}\right)\right) \\
& u_{n+1}(x, t)=\sum_{k=1}^{n} B_{k} \bar{\phi}_{k}(x, t) .
\end{aligned}
$$

Lemma 3.1 If $u_{n}(x, t) \stackrel{\|\cdot\|_{W_{2}^{(1,1)}}}{\longrightarrow} \bar{u}(x, t)(n \rightarrow \infty)$ and $\left(x_{n}, t_{n}\right) \rightarrow(y, z)$, then $u_{n}\left(x_{n}, t_{n}\right) \rightarrow \bar{u}(y, z)(n \rightarrow \infty)$

Proof We have

$$
\begin{aligned}
\left|u_{n}\left(x_{n}, t_{n}\right)-\bar{u}(y, z)\right|= & \mid u_{n}\left(x_{n}, t_{n}\right)-u_{n}(y, z)+u_{n}(y, z) \\
& -\bar{u}(y, z) \mid
\end{aligned}
$$

$$
\leq\left|u_{n}\left(x_{n}, t_{n}\right)-u_{n}(y, z)\right|+\left|u_{n}(y, z)-\bar{u}(y, z)\right| .
$$

For absolute first on the right side of the above inequality, we have

$$
\left|u_{n}\left(x_{n}, t_{n}\right)-u_{n}(y, z)\right|=\left|\left\langle u_{n}(x, t), K_{\left(x_{n}, t_{n}\right)}(x, t)-K_{(y, z)}(x, t)\right\rangle_{W_{2}^{(1,1)}}\right|
$$

$$
\leq\left\|u_{n}(x, t)\right\|_{W_{2}^{(1,1)}}\left\|K_{\left(x_{n}, t_{n}\right)}(x, t)-K_{(y, z)}(x, t)\right\|_{W_{2}^{(1,1)}} .
$$

From the convergence of sequence $\left\{u_{n}(x, t)\right\}_{n=1}^{\infty}$, we conclude that there exists a constant $N$, such that

$\left\|u_{n}(x, t)\right\|_{W_{2}^{(1,1)}} \leq N\|\bar{u}(x, t)\|_{W_{2}^{(1,1)}}$

when $n \geq N$. At the same time, it can be proved that

$$
\left\|K_{\left(x_{n}, t_{n}\right)}(x, t)-K_{(y, z)}(x, t)\right\|_{W_{2}^{(1,1)}} \rightarrow 0
$$

when $n \rightarrow \infty$. Thus, $\left|u_{n}\left(x_{n}, t_{n}\right)-u_{n}(y, z)\right| \rightarrow 0$ when $\left(x_{n}, t_{n}\right) \rightarrow(y, z)$. From $\|u\|_{c} \leq M\|u\|_{W_{2}^{(1,1)}}$ for any $(y, z) \in D$, it holds that

$$
\left\|u_{n}(y, z)-\bar{u}(y, z)\right\|_{c} \rightarrow 0 \quad(n \rightarrow \infty)
$$

when

$$
\left\|u_{n}(y, z)-\bar{u}(y, z)\right\|_{W_{2}^{(1,1)}} \rightarrow 0 \quad(n \rightarrow \infty) .
$$

Therefore, here, we conclude that

$u_{n}\left(x_{n}, t_{n}\right) \rightarrow \bar{u}(y, z) \quad(n \rightarrow \infty)$

when $\left(x_{n}, t_{n}\right) \rightarrow(y, z)[9] . \diamond$.

Using the continuation of $N($.$) , it will be obtained that$ $N\left(u_{n}\left(x_{n}, t_{n}\right)\right) \rightarrow N(\bar{u}(y, z))$ when $n \rightarrow \infty$. In addition, this shows that

$$
F u_{n}\left(x_{n}, t_{n}\right) \rightarrow F \bar{u}(y, z)(n \rightarrow \infty) \text {. }
$$

From the method listed above, convergence theorem will be obtained.

Theorem 3.1 Suppose that the sequence $\left\{\left\|u_{n}(x, t)\right\|_{W_{2}^{(1,1)}}\right\}_{n=1}^{\infty}$ is bounded in (3), if $\left\{\left(x_{i}, t_{i}\right)\right\}_{i=1}^{\infty}$ is dense in $D$, then $n$-term approximate solution $u_{n}(x, t)$ converges to the exact solution $u(x, t)$ of Eq. (1) and exact solution is expressed as

$$
u(x, t)=\sum_{i=1}^{\infty} B_{i} \bar{\phi}_{i}(x, t)
$$

where $B_{i}$ is given by (4).

Proof The theorem is proved in three steps:

(a) At this step, we provide that the sequence $\left\{u_{n}(x, t)\right\}_{n=1}^{\infty}$ with formula (3) is converged. For this purpose from (4), we have 
$u_{n+1}(x, t)=u_{n}(x, t)+B_{n} \bar{\phi}_{n}(x, t)$.

From recent equality and using the orthogonality of $\left\{\bar{\phi}_{i}(x, t)\right\}_{i=1}^{\infty}$, it follows

$\left\|u_{n+1}(x, t)\right\|_{W_{2}^{(1,1)}}^{2}=\left\|u_{n}(x, t)\right\|_{W_{2}^{(1,1)}}^{2}+B_{n}^{2}$.

Thus, sequence $\left\{\left\|u_{n}(x, t)\right\|_{W_{2}^{(1,1)}}\right\}_{n=1}^{\infty}$ is monotone increasing. In other hand, sequence $\left\{\left\|u_{n}(x, t)\right\|_{W_{2}^{(1,1)}}\right\}_{n=1}^{\infty}$ is bounded; therefore, this sequence is convergent. Therefore, there exists a constant $c$, such that

$\sum_{i=1}^{\infty} B_{i}^{2}=c$

This illustrate that

$B_{i}=\sum_{k=1}^{i} \alpha_{i k}\left(f\left(x_{k}, t_{k}\right)+F u_{i}\left(x_{k}, t_{k}\right)\right) \in \ell^{2} \quad(i=1,2, \ldots)$.

Using the orthogonality of $\left\{u_{n+1}(x, t)-u_{n}(x, t)\right\}_{n=1}^{\infty}$ if $m>n$, then

$$
\begin{aligned}
& \left\|u_{m}(x, t)-u_{n}(x, t)\right\|_{W_{2}^{(1,1)}}^{2}=\| u_{m}(x, t)-u_{m-1}(x, t) \\
& +u_{m-1}(x, t)-u_{m-2}(x, t)+\cdots+u_{n+1}(x, t)-u_{n}(x, t) \|_{W_{2}^{(1,1)}}^{2} \\
& =\left\|u_{m}(x, t)-u_{m-1}(x, t)\right\|_{W_{2}^{(1,1)}}^{2}+\cdots \\
& \quad+\left\|u_{n+1}(x, t)-u_{n}(x, t)\right\|_{W_{2}^{(1,1)}}^{2} \\
& \quad \sum_{i=n+1}^{m} B_{i}^{2} \rightarrow 0 \quad(n \rightarrow \infty) .
\end{aligned}
$$

Considering that $W_{2}^{(1,1)}(D)$ is complete, so it follows

$u_{n}(x, t) \stackrel{\|\cdot\|_{W_{2}^{(1,1)}}}{\longrightarrow} \bar{u}(x, t) \quad(n \rightarrow \infty)$

Therefore

$$
\bar{u}(x, t)=\sum_{i=1}^{\infty} B_{i} \bar{\phi}_{i}(x, t)
$$

(b) We define the projection operator as follows:

$$
P_{n} \bar{u}(x, t)=\sum_{i=1}^{n} B_{i} \bar{\phi}_{i}(x, t) .
$$

Thus

$$
u_{n+1}(x, t)=P_{n} \bar{u}(x, t) .
$$

We claim

$u_{n+1}\left(x_{k}, t_{k}\right)=\bar{u}\left(x_{k}, t_{k}\right), k \leq n$.

To this end, we have $u_{n+1}\left(x_{k}, t_{k}\right)=\left\langle u_{n+1}(x, t), \phi_{k}(x, t)\right\rangle_{W_{2}^{(1,1)}}$

$=\left\langle P_{n} \bar{u}(x, t), \phi_{k}(x, t)\right\rangle_{W_{2}^{(1,1)}}$

$=\left\langle\bar{u}(x, t), P_{n} \phi_{k}(x, t)\right\rangle_{W_{2}^{(1,1)}}$

$=\left\langle\bar{u}(x, t), \phi_{k}(x, t)\right\rangle_{W_{2}^{(1,1)}}$

$=\bar{u}\left(x_{k}, t_{k}\right)$

Hence

$F u_{n+1}\left(x_{k}, t_{k}\right)=F \bar{u}\left(x_{k}, t_{k}\right), \quad k \leq n$.

(c) It is obvious that $\bar{u}(x, t)$ is the solution of Eq. (1). From (6), it follows

$\bar{u}\left(x_{j}, t_{j}\right)=\sum_{i=1}^{\infty} B_{i}\left\langle\bar{\phi}_{i}(x, t), \phi_{j}(x, t)\right\rangle_{W_{2}^{(1,1)}}$.

By multiplying both sides of (7) by $\alpha_{n j}$ and summing for $j$ from 1 to $n$, we have

$$
\begin{aligned}
& \sum_{j=1}^{n} \alpha_{n j} \bar{u}\left(x_{j}, t_{j}\right)=\sum_{i=1}^{\infty} B_{i}\left\langle\bar{\phi}_{i}(x, t), \sum_{j=1}^{n} \alpha_{n j} \phi_{j}(x, t)\right\rangle_{W_{2}^{(1,1)}} \\
& =\sum_{i=1}^{\infty} B_{i}\left\langle\bar{\phi}_{i}(x, t), \bar{\phi}_{n}(x, t)\right\rangle_{W_{2}^{(1,1)}}=B_{n} .
\end{aligned}
$$

From (8) and (2), if $n=1$, then

$$
\begin{aligned}
& \alpha_{11} \bar{u}\left(x_{1}, t_{1}\right)=B_{1}=\alpha_{11}\left(f\left(x_{1}, t_{1}\right)+F u_{1}\left(x_{1}, t_{1}\right)\right) . \\
& \text { If } n=2 \text {, then } \\
& \quad \alpha_{21} \bar{u}\left(x_{1}, t_{1}\right)+\alpha_{22} \bar{u}\left(x_{2}, t_{2}\right)=B_{2} \\
& =\alpha_{21}\left(f\left(x_{1}, t_{1}\right)+F u_{2}\left(x_{1}, t_{1}\right)\right)+\alpha_{22}\left(f\left(x_{2}, t_{2}\right)+F u_{2}\left(x_{2}, t_{2}\right)\right) \\
& =\alpha_{21}\left(f\left(x_{1}, t_{1}\right)+F \bar{u}_{2}\left(x_{1}, t_{1}\right)\right)+\alpha_{22}\left(f\left(x_{2}, t_{2}\right)+F u_{2}\left(x_{2}, t_{2}\right)\right) \\
& \left.=\alpha_{21} \bar{u}\left(x_{1}, t_{1}\right)\right)+\alpha_{22}\left(f\left(x_{2}, t_{2}\right)+F u_{2}\left(x_{2}, t_{2}\right)\right) \text {. From the }
\end{aligned}
$$
above equality, it follows

$$
\bar{u}\left(x_{2}, t_{2}\right)=f\left(x_{2}, t_{2}\right)+F u_{2}\left(x_{2}, t_{2}\right) .
$$

In the same way, we obtain that

$\bar{u}\left(x_{n}, t_{n}\right)=f\left(x_{n}, t_{n}\right)+F u_{n}\left(x_{n}, t_{n}\right)$.

Since $\left\{\left(x_{i}, t_{i}\right)\right\}_{i=1}^{\infty}$ is dense in $D$, therefore, for any $(y, z) \in D$, there exists a subsequence $\left\{\left(x_{n_{k}}, t_{n_{k}}\right)\right\}_{k=1}^{\infty}$ converging to $(y, z)$. From Lemma 3.1 and above equality, we obtain that

$\bar{u}(y, z)=f(y, z)+F \bar{u}(y, z)$.

Thus, $\bar{u}(x, t)$ is the solution of Eq. (1) and

$u(x, t)=\sum_{i=1}^{\infty} B_{i} \bar{\phi}_{i}(x, t)$. 
where

Theorem 3.2 Suppose $u(x, t)$ is the solution of Eq. (1) and $r_{n}(x, t)$ is the error in the approximate solution $u_{n+1}(x, t)$, where $u_{n+1}(x, t)$ is given by (3). Then, $r_{n}(x, t)$ is monotone decreasing in the sense of $\|\cdot\|_{W_{2}^{(1,1)}}$.

Proof If the $u(x, t)$ and $u_{n+1}(x, t)$ are, respectively, functions in (1) and (6), in this case, we have

$$
\begin{aligned}
& \left\|r_{n}(x, t)\right\|_{W_{2}^{(1,1)}}^{2}=\left\|u(x, t)-u_{n+1}(x, t)\right\|_{W_{2}^{(1,1)}}^{2} \\
& =\left\|\sum_{i=n+1}^{\infty} B_{i} \bar{\phi}_{i}(x, t)\right\|_{W_{2}^{(1,1)}}^{2}=\sum_{i=n+1}^{\infty} B_{i}^{2} .
\end{aligned}
$$

This illustrate that the error $r_{n}(x, t)$ is monotone decreasing in the sense of $\|\cdot\|_{W_{2}^{(1,1)}}$ [9].

\section{Numerical examples}

We implement the method presented in this article for some examples. We obtain absolute error of the approximate solution values in the selected points $(x, t)=\left(\frac{1}{2^{i}}, \frac{1}{2^{i}}\right)(i=1,2, \ldots, 6)$. Of course, the choice of points is completely customized. Examples of references listed have been selected, so that we can compare the results in here with results in its references.

Example 4.1 [7] Consider the following nonlinear twodimensional Volterra integral equation:

$u(x, t)=f(x, t)+\int_{0}^{t} \int_{0}^{x}\left(x y^{2}+\cos (z)\right) u^{2}(y, z) \mathrm{d} y \mathrm{~d} z \quad 0 \leq x, t \leq 1$

where

$f(x, t)=x \sin (t)\left(1-\frac{1}{9} x^{2} \sin ^{2}(t)\right)+\frac{1}{10} x^{6}\left(\frac{1}{2} \sin (2 t)-t\right)$.

The exact solution is $u(x, t)=x \sin (t)$. Table 1 illustrates the numerical results for this example. In addition, for points $\left(x_{i}, t_{i}\right)=(0.001 i, 0.001 i) \quad(i=1,2, \ldots, 1000)$, the maximum error is as follows:

$M=\max _{i=1,2, \ldots, 1000}\left\{\left|r\left(x_{i}, t_{i}\right)\right|\right\}=0.04873005644$

Example 4.2 [7] Consider the following nonlinear twodimensional Volterra integral equation:

$u(x, t)=f(x, t)+\int_{0}^{t} \int_{0}^{x}(x+t-z-y) u^{2}(y, z) \mathrm{d} y \mathrm{~d} z \quad 0 \leq x, t \leq 1$

where

$f(x, t)=x+t-\frac{1}{12} x t\left(x^{3}+4 x^{2} t+4 x t^{2}+t^{3}\right)$.

The exact solution is $u(x, t)=x+t$. Table 2 illustrates the numerical results for this example. In addition, for points $\left(x_{i}, t_{i}\right)=(0.001 i, 0.001 i)(i=1,2, \ldots, 1000)$, the maximum error is as follows:

$M=\max _{i=1,2, \ldots, 1000}\left\{\left|r\left(x_{i}, t_{i}\right)\right|\right\}=0.165027043$

where

$r(x, t)=u(x, t)-u_{40}(x, t)$.

Example 4.3 [8] Consider the following nonlinear twodimensional Volterra integral equation:

$u(x, t)=f(x, t)+\int_{0}^{t} \int_{0}^{x} u^{2}(y, z) \mathrm{d} y \mathrm{~d} z \quad(x, t) \in[0,1) \times[0,1)$

where

$f(x, t)=x^{2}+t^{2}-\frac{1}{45} x t\left(9 x^{4}+10 x^{2} t^{2}+9 t^{4}\right)$.

The exact solution of this problem is $u(x, t)=x^{2}+t^{2}$. Table 3 illustrates the numerical results for this example. In addition, for points $\left(x_{i}, t_{i}\right)=(0.001 i, 0.001 i)(i=1,2$, $\ldots, 1000)$, the maximum error is as follows:

$M=\max _{i=1,2, \ldots, 1000}\left\{\left|r\left(x_{i}, t_{i}\right)\right|\right\}=0.3099048997$

where

$r(x, t)=u(x, t)-u_{30}(x, t)$.

Table 1 Absolute error of approximate solution for Example 4.1

\begin{tabular}{lllll}
\hline Node & Exact solution $u(x, t)$ & Approximate $u_{30}(x, t)$ & Absolute error in presented method & Absolute error in [7] with $\alpha=4(m=32)$ \\
\hline$\left(\frac{1}{2}, \frac{1}{2}\right)$ & 0.23972 & 0.23978 & 0.00006 & $1.4 \times 10^{-2}$ \\
$\left(\frac{1}{4}, \frac{1}{4}\right)$ & 0.061850 & 0.061979 & 0.000129 & $7.9 \times 10^{-3}$ \\
$\left(\frac{1}{8}, \frac{1}{8}\right)$ & 0.015584 & 0.015654 & 0.000070 & $4.1 \times 10^{-3}$ \\
$\left(\frac{1}{16}, \frac{1}{16}\right)$ & 0.0039037 & 0.003957 & 0.0000533 & $2.2 \times 10^{-3}$ \\
$\left(\frac{1}{32}, \frac{1}{32}\right)$ & 0.00097641 & 0.001036 & 0.00005959 & $1.2 \times 10^{-3}$ \\
$\left(\frac{1}{64}, \frac{1}{64}\right)$ & 0.00024412 & 0.000990 & 0.00074588 & $9.3 \times 10^{-9}$ \\
\hline
\end{tabular}


Table 2 Absolute error of approximate solution for Example 4.2

\begin{tabular}{lllll}
\hline Node & Exact solution $u(x, t)$ & Approximate $u_{30}(x, t)$ & Absolute error in presented method & Absolute error in [7] with $\alpha=4(m=32)$ \\
\hline$\left(\frac{1}{2}, \frac{1}{2}\right)$ & 1 & 0.99734 & 0.00266 & $3.1 \times 10^{-2}$ \\
$\left(\frac{1}{4}, \frac{1}{4}\right)$ & 0.50000 & 0.49989 & 0.00011 & $3.1 \times 10^{-2}$ \\
$\left(\frac{1}{8}, \frac{1}{8}\right)$ & 0.25000 & 0.24995 & 0.00005 & $3.1 \times 10^{-2}$ \\
$\left(\frac{1}{16}, \frac{1}{16}\right)$ & 0.12500 & 0.12490 & 0.000101 & $3.1 \times 10^{-2}$ \\
$\left(\frac{1}{32}, \frac{1}{32}\right)$ & 0.062500 & 0.062375 & 0.000125 & $3.1 \times 10^{-2}$ \\
$\left(\frac{1}{64}, \frac{1}{64}\right)$ & 0.031250 & 0.047954 & 0.016704 & $2.2 \times 10^{-9}$ \\
\hline
\end{tabular}

Table 3 Absolute error of approximate solution for Example 4.3

\begin{tabular}{lllll}
\hline Node & Exact solution $u(x, t)$ & Approximate $u_{30}(x, t)$ & Absolute error in presented method & Absolute error in [8] with $m=32$ \\
\hline$\left(\frac{1}{2}, \frac{1}{2}\right)$ & 0.50000 & 0.49736 & 0.00264 & $1.1 D-2$ \\
$\left(\frac{1}{4}, \frac{1}{4}\right)$ & 0.12500 & 0.12524 & 0.00024 & $6.4 D-4$ \\
$\left(\frac{1}{8}, \frac{1}{8}\right)$ & 0.031250 & 0.031400 & 0.000150 & $2.5 D-3$ \\
$\left(\frac{1}{16}, \frac{1}{16}\right)$ & 0.0078125 & 0.007907 & 0.0000945 & $3.5 D-3$ \\
$\left(\frac{1}{32}, \frac{1}{32}\right)$ & 0.0019531 & 0.002090 & 0.0001369 & $3.5 D-4$ \\
$\left(\frac{1}{64}, \frac{1}{64}\right)$ & 0.00048828 & 0.002007 & 0.0015187 & $4.3 D-5$ \\
\hline
\end{tabular}

\section{Conclusion and comments}

This paper deals with a computational method for approximate solution of Volterra integral equations of the second kind based on the expansion of the solution as series of reproducing kernel functions. The advantage of the present method (compared with methods based on basis sets of different kinds) is not limitation on the nonlinear term. Considering that absolute errors of approximate solution in given points are small enough, so it follows that the presentation method in this article is right. The codes were written in Maple. We think that this method can be generalized to the new inner multiply that it provides reproducing kernel space.

Open Access This article is distributed under the terms of the Creative Commons Attribution 4.0 International License (http://crea tivecommons.org/licenses/by/4.0/), which permits unrestricted use, distribution, and reproduction in any medium, provided you give appropriate credit to the original author(s) and the source, provide a link to the Creative Commons license, and indicate if changes were made.

\section{References}

1. Farengo, R., Lee, Y.C., Guzdar, P.N.: An electromagnetic integral equation: application to microtearing modes. Phys. Fluids. 26, 3515-3523 (1983)
2. Mckee, S., Tang, T., Diogo, T.: An Euler-type method for twodimensional Volterra integral equations of the first kind. IMA J. Numer. Anal. 20, 423-440 (2000)

3. Graham, I.G.: Collocation method for two-dimensional weakly singular integral equations. J. Aust. Math. Soc. (Ser. B) 22, 456-473 (1981)

4. Hanson, R.J., Phillips, J.L.: Numerical solution of two-dimensional integral equations using linear elements. SIAM J. Numer. Anal. 15(1), 113-121 (1978)

5. Mirzaee, F., Rafei, Z.: The block by block method for the numerical solution of the nonlinear two-dimensional Volterra integral equations. J. King Saud Univ. Sci. 23, 191-195 (2011)

6. Mirzaee, F., Hoseini, A.A.: A computational method based on hybrid of block-pulse functions and Taylor series for solving twodimensional nonlinear integral equations. Alex. Eng. J. 53, 185-190 (2014)

7. Babolian, E., Bazm, S., Lima, P.: Numerical solution of nonlinear two-dimensional integral equations using rationalized Harr functions. Commun. Nonlinear Sci. Numer. Simul. 16, 1164-1175 (2011)

8. Maleknejad, K., Sohrabi, B.B.: Two-dimensional FCBS: application to nonlinear Volterra integral equations. In: Proceedings of the World Congress on Engineering (WCE), vol II, 1-3 July 2009, London

9. Cui, M., Lin, Y.: Nonlinear Numerical Analysis in the Reproducing Kernel Space. Nova Science Publishers, New York (2008) 